This item was submitted to Loughborough's Research Repository by the author.

Items in Figshare are protected by copyright, with all rights reserved, unless otherwise indicated.

\title{
Numerical simulation of oxy-fuel jet flames using unstructured LES-CMC
}

PLEASE CITE THE PUBLISHED VERSION

http://dx.doi.org/10.1016/j.proci.2014.05.032

PUBLISHER

(c) Elsevier

VERSION

AM (Accepted Manuscript)

PUBLISHER STATEMENT

This work is made available according to the conditions of the Creative Commons Attribution-NonCommercialNoDerivatives 4.0 International (CC BY-NC-ND 4.0) licence. Full details of this licence are available at: https://creativecommons.org/licenses/by-nc-nd/4.0/

\section{LICENCE}

CC BY-NC-ND 4.0

\section{REPOSITORY RECORD}

Garmory, Andrew, and E. Mastorakos. 2014. "Numerical Simulation of Oxy-fuel Jet Flames Using Unstructured LES-CMC". Loughborough University. https://hdl.handle.net/2134/17319. 


\title{
Numerical Simulation of Oxy-Fuel Jet Flames Using Unstructured LES-CMC
}

\author{
A. Garmory ${ }^{\mathrm{a}, *}$, E. Mastorakos ${ }^{\mathrm{b}}$ \\ ${ }^{a}$ Department of Aeronautical and Automotive Engineering, Loughborough University, \\ Loughborough, LE11 3TU, United Kingdom \\ ${ }^{b}$ Hopkinson Laboratory, Department of Engineering, University of Cambridge, \\ Cambridge CB2 1PZ, United Kingdom
}

\begin{abstract}
A finite volume Conditional Moment Closure (CMC) formulation has been developed as an LES sub-grid combustion model. This allows unstructured meshes to be used for both LES and CMC grids making the method more applicable to complex geometry. The method has been applied to an oxy-fuel jet flame. This flame offers new challenges to combustion modelling due to a high $\mathrm{CO}_{2}$ content in the oxidiser stream and significant $\mathrm{H}_{2}$ content in the fuel stream. The density ratio of the two streams is of the order 5 and the viscosity of the two streams will also differ. All the flames simulated showed localised extinction in the region around 3-5 jet diameters downstream of the nozzle, which is in very good agreement with the experiment. Trends for conditional and unconditional statistics with changing levels of $H_{2}$ in the fuel are correctly captured by the LES-CMC method, although different levels of agreement are observed for different species and temperature and possible reasons for this are discussed. The degree of extinction is also correctly
\end{abstract}

\footnotetext{
${ }^{*}$ Corresponding author

Email address: a.garmory@lboro.ac.uk (A. Garmory)
} 
predicted to increase as the $H_{2}$ content of the jet is reduced, showing the ability of the CMC method to predict complex turbulence-chemistry interaction phenomenon in the presence of changing fuel composition.

Keywords:

LES, CMC, extinction, Oxy-fuel, non-premixed

\section{Introduction}

As a means of mitigating the environmental effects of combustion there is currently much interest in developing carbon capture and storage (CCS) technologies. To facilitate the separation and hence removal of $\mathrm{CO}_{2}$ from combustion products it is desirable that the only products should be $\mathrm{CO}_{2}$ and water vapour. This also has the advantage of removing $N O_{x}$ emissions. Removing the nitrogen from the oxidiser is one method to achieve this; however it has been found that the very high flame temperature in this pure oxygen arrangement is undesirable [1]. Therefore there is interest in diluting the oxider stream with carbon dioxide so as to reduce the flame temperature. Due to the different properties of $\mathrm{N}_{2}$ and $\mathrm{CO}_{2}$, a $\mathrm{CO}_{2}$ diluted oxy-fuel flame will behave in a different manner to an air-fuel flame; becoming more prone to extinction [2] with the consequence that extra stabilisation mechanisms such as enriching the fuel stream with hydrogen may be necessary. The changed operating conditions present new challenges to computational modelling. Here we have applied the Conditional Moment Closure (CMC) combustion model to an oxy-fuel jet which was the subject of a recent in depth experimental investigation into the behaviour of the early part of the jet [3]. The flame has a different composition to a standard methane-air flame 
and the 'A-series' of flames were observed to exhibit an increasing degree of extinction as the level of $\mathrm{H}_{2}$ in the fuel stream was reduced. Our aim is to assess the ability of the LES-CMC method to capture this trend.

Much effort has been focussed in recent years on the development of Large Eddy Simulation (LES) for both pre and non-premixed combustion. This development has been successful in capturing unsteady behaviour, such as transient localised extinctions. The Eulerian Stochastic Fields [4] and Flamelet/Progress Variable (FPV) [5] methods have been used to predict extinction in the Sandia piloted jet flames. The Multiple Mapping Closure (MMC) method, which can be thought of as combining elements of PDF and CMC modelling and is able to predict partial extinctions, has also been successfully applied to the Sandia flames [6]. A hybrid mesh-particle LES/PDF formulation has been successful in reproducing the extinction and reignition behaviour seen in a DNS calculation of a $\mathrm{CO} / \mathrm{H}_{2}$ planar jet [7].

The LES-CMC model has also been successfully applied to the Sandia piloted flames [8], giving similar predictions of extinction to the Stochastic Fields [4] and MMC [6] methods. It has also been successfully applied to other gaseous [9] and spray [10] flames. It has been seen that the combination of transport and scalar dissipation rate terms are able to capture transient extinction and reignition. These results have shown the ability of the CMC method to capture complex phenomenon such as extinction or iginition with complex chemistry at a relatively low computational cost. A drawback of the existing $\mathrm{CMC}$ formulation is that the finite difference scheme used does not lend itself to complex geometries or to localised grid refinement. This paper, therefore, presents results using a new finite volume implementation 
of the CMC equations which allows for an unstructured CMC grid to be used offering greater potential as a CFD tool.

\section{Formulation}

\subsection{Unstructured LES-CMC Formulation}

The principle behind the LES-CMC method for non-premixed combustion is that if the local filtered density function, $\widetilde{\mathcal{P}}(\eta)$, of mixture fraction is known and the conditional average $Q_{\phi}=\widetilde{\phi \mid \eta}$ is known for a scalar $\phi$ then the unconditional local filtered value of the scalar can be found from $\widetilde{\phi}=\int_{0}^{1} \widetilde{\phi \mid \eta} \widetilde{\mathcal{P}}(\eta) d \eta$. A transport equation for $Q$ can then be derived with the advantage that for much of the flow the gradients of $Q$ can be expected to be much less steep than $\widetilde{\phi}$ and so a far coarser grid can be used. By solving a transport equation for $Q$ the conditional average in a cell can take values which will give an extinguished composition at stoichiometry, therefore predicting a localised extinction. The size of the extinction is determined by the CMC cell size, so if the CMC grid can be refined in a region where localised extinctions are expected then this will lead to more accurate prediction of localised extinction. As such an unstructured CMC grid capable of local refinement would be advantageous.

In this work we have used a conservative finite-volume formulation of the $\mathrm{CMC}$ equation. A conservative form of the $\mathrm{CMC}$ equation has been previously derived in the RANS context [11], however this requires the flux of $\bar{\rho} \widetilde{\mathcal{P}}(\eta)$ to be calculated in all transport terms. This can be numerically difficult to implement and so we have started from the finite difference CMC equation that has previously been used in LES. The CMC equation as used 
with LES can be derived by filtering the transport equations for the reactive scalars [12]. Using the primary closure assumption, the CMC equation becomes

$$
\frac{\partial Q}{\partial t}+\widetilde{u_{i} \mid \eta} \frac{\partial Q}{\partial x_{i}}=\widetilde{N \mid \eta} \frac{\partial^{2} Q}{\partial \eta^{2}}+\widetilde{\omega \mid \eta}+e_{f}
$$

where $\widetilde{u_{i} \mid \eta}$ is the conditionally filtered velocity, $\widetilde{N \mid \eta}$ is the conditionally filtered scalar dissipation rate, $\widetilde{\omega \mid \eta}$ is the conditionally filtered reaction rate, while the term $e_{f}$ represents the sub-grid scale conditional transport. To produce an unstructured $\mathrm{CMC}$ formulation it is necessary to recast Eq. (1) into finite volume form. The first stage in doing this gives

$$
\frac{\partial Q}{\partial t}+\nabla \cdot(\widetilde{Q \boldsymbol{u} \mid \eta})=Q \nabla \cdot(\widetilde{\boldsymbol{u} \mid \eta})+\widetilde{N \mid \eta} \frac{\partial^{2} Q}{\partial \eta^{2}}+\widetilde{\omega \mid \eta}+e_{f}
$$

Two terms are worthy of further note here. An extra convection term, $Q \nabla$. $\widetilde{(\boldsymbol{u} \mid \eta)}$, appears. If $\widetilde{\boldsymbol{u} \mid \eta}$ is solenoidal this term will disappear but, as the conditional velocity does not have to satisfy this requirement, it will be left here. The term $e_{f}$ representing the sub-grid conditional flux needs to be modelled. The model used in finite difference implementations [13] is not conservative and hence not suitable for the finite volume formulation. Here we choose to model the term with a simple turbulent diffusion term, $\nabla$. $\left(D_{t} \nabla Q\right)$. The influence of this term is a subject for further research. However we note that previous work [14] has shown the influence of the $e_{f}$ term to be minor compared to the other terms. The final form of the CMC equation used in this work in integral form is 


$$
\begin{aligned}
\frac{\partial Q}{\partial t} & +\frac{1}{V} \oint(\widetilde{(\boldsymbol{u} \mid \eta}) \cdot d \boldsymbol{A}=\frac{1}{V} \oint\left(D_{t} \nabla Q\right) \cdot d \boldsymbol{A} \\
& \left.+\frac{Q}{V} \oint \widetilde{(\boldsymbol{u} \mid \eta}\right) \cdot d \boldsymbol{A}+\widetilde{N \mid \eta} \frac{\partial^{2} Q}{\partial \eta^{2}}+\widetilde{\dot{w} \mid \eta}
\end{aligned}
$$

In the extra convection term $Q$ has been taken as constant throughout the CMC control volume, $V$, and so can be taken outside the integral.

\subsection{Unstructured LES-CMC Coupling Strategy}

In order to implement Eq. (3) in an LES calculation the momentum and mixture fraction field need to be calculated on a fine LES grid while the conditional averages are calculated on a coarser CMC grid. During one timestep both LES and CMC need to be updated with information being exchanged between the two grids to enable this. As in previous implementations, each

LES cell must be associated with a CMC cell such that if $\widetilde{\mathcal{P}}(\eta)$ is known for the LES cell then the resolved temperature and density for that LES cell can be found from the CMC cell conditional averages. The $\widetilde{N \mid \eta}$ term can be calculated by averaging over the LES cells associated with one CMC cell.

Previously the same method was used to find an averaged conditional velocity for each CMC cell. However, in the new unstructured formulation a different method is employed. A list of CMC cell 'centres' (which can be generated by widely available unstructured meshing packages) are read in to the LES-CMC solver and the LES cells are allocated to their nearest CMC centre. We then go a step further by saying that the collection of LES cells mapped to a CMC cell become the $\mathrm{CMC}$ cell so that the faces of the $\mathrm{CMC}$ cell are the faces of the LES cells at the edge of the CMC cell. We follow the 
successful previous practice in LES-CMC [14] of modelling the conditional velocity as equal to the unconditional velocity, $\widetilde{\boldsymbol{u} \mid \eta}=\widetilde{\boldsymbol{u}}$.

For the convection term on the LHS of Eq. (3) the flux $Q \boldsymbol{u} \cdot \boldsymbol{A}$ needs to be evaluated over the surface of the CMC cell. This surface is made up of LES cell surfaces and hence a $Q_{f}$ face value and a volume flux is needed for each LES/CMC face. $Q_{f}$ is found by a suitable discretisation scheme for each LES/CMC face. The scheme used for this work is first-order upwind although higher order schemes could be used to find $Q_{f}$. The unstructured grid formulation allows for greater grid refinement where required, which will reduce the discretisation error. In any unstructured LES code the volume flux, $\boldsymbol{u} \cdot \boldsymbol{A}$, across each LES face must already be calculated and hence it is logical to use this to calculate the conditional flux between CMC cells. The advantages of this method are that not only does it eliminate the need to average the LES velocity field, but that more detail from the LES velocity field can be included in the CMC transport. Flow structures smaller than the CMC grid but larger than the LES resolution are no longer filtered out of the CMC transport in the same way.

The extra convection term on the RHS of Eq. (3) is evaluated by using the LES face fluxes $\boldsymbol{u} \cdot \boldsymbol{A}$ weighted by the cell value of $Q$ for the cell which the rate of change is being evaluated for. This is equivalent to taking the value of $Q$ as being constant over the control volume when converting from Eq. (1) to Eq. (2), which is an assumption also used when finding the unconditional values on the LES grid using the conditional values for the larger CMC cell.

The conditional diffusion term is calculated in a similar manner; $D_{t} \boldsymbol{A}$ is taken from the LES solver and the gradient of $\mathrm{Q}$ is calculated for each 
LES/CMC face using the CMC grid values. Here we have chosen to take the value of $D_{t}$ based on the LES scale using the argument that the sub-LES grid motion will be responsible for the transport not included in the convection term. This is a modelling choice, but as noted earlier previous work [14] has shown the influence of the diffusion term to be minor compared to the other terms.

The unstructured CMC formulation developed here is designed so that it can be called as subroutines from a generic 'host' finite volume CFD code. These subroutines can be placed where required to initialise the mapping of CMC and LES cells and then in the main timestepping loop to pass flow field information to the CMC and unconditional values back to the LES solver. An extra CMC mesh is required which can be created by existing meshing packages and converted into a suitable cell centre position text file. There is no restriction on the CMC cell shape as they will be made up of a collection of LES cells. The CMC cell centres are initially partitioned using the same domain decomposition as for the LES grid allowing parallisation of the CMC calculation. Note that a CMC cell is located entirely on one processor. As the grid refinement for the LES and CMC cells will not necessarily be needed in same place, the integration of chemistry for the cells is redistributed over the processors for load balancing.

\subsection{Simulation Details}

The oxy-fuel jet of [3] consists of a $d=5 \mathrm{~mm} \mathrm{CH}_{4} / \mathrm{H}_{2}$ jet surrounded by a $\mathrm{CO}_{2} / \mathrm{O}_{2}$ co-flow. This computational domain representing this for both LES and CMC is conical in shape being 10d in diameter at the jet inlet plane and $24 \mathrm{~d}$ at the outlet plane which is $80 \mathrm{~d}$ downstream. The base and sides of 
the cone are set as inlets and the outlet plane is set as a pressure outlet where the pressure is set to atmospheric and all other variables have a zero gradient boundary condition. The size and shape of the domain should ensure that the effect of the boundaries on the early part of the jet is minimised. The LES mesh has 2.3M hexahedral elements in an O-ring arrangement using 60 cells across the jet nozzle. The CMC grid has been designed using the same O-ring arrangement but with a reduced number of cells, 29k, using 15 CMC cells across the jet nozzle. The highest density of CMC cells is found in the shear layers in the early part of the jet. Far downstream and away from the jet axis the conditional values will not have steep gradients and so large CMC cells can be used here. Both LES and CMC grids are shown in supplementary material. This o-ring mesh with localised refinement has been made possible with the new unstructured formulation.

The LES equations are solved using the OpenFOAM [15] code. The velocity field is solved using a 'Pimple' pressure correction algorithm. This is a hybrid SIMPLE/PISO scheme used in the standard OpenFOAM release combustion solvers [15]. At the end of the timestep the CMC solver is called with scalar dissipation rate and LES/CMC face flux data to allow the $Q$ values to be updated. A second routine is then used to return the local LES density and molecular viscosity given the mean and variance of mixture fraction for that cell. The constant Smagorinsky model [16], with constant equal to 0.14 using the standard settings of the OpenFOAM implemenatation of the model, has been applied for the sub-grid stress. The FDF of mixture fraction is found from the resolved and estimated sub-grid variance assuming a $\beta$ function. The mean resolved mixture fraction $\tilde{\xi}$ is calculated by the LES 
solver with molecular and turbulent Schmidt numbers equal to 0.7. The variance is calculated from $\widetilde{\xi^{\prime \prime 2}}=C_{v} \Delta^{2}\left(\frac{\partial \tilde{\xi}}{\partial x_{i}}\right)$ where $\Delta$ is the filter width. The constant $C_{v}$ is set to 0.1 following [17].

For the CMC code the conditional scalar dissipation rate is found from the resolved and sub-grid variance of mixture fraction in the CMC cell and the averaged scalar dissipation rate of the LES cells within one CMC cell. The Amplitude Mapping Closure (AMC) model [18] is then applied to find $\widetilde{N \mid \eta}$. The scalar dissipation rate in each LES cell is made up of the resolved component $\tilde{N}_{\text {res }}=D\left(\frac{\partial \widetilde{\xi}}{\partial x_{i}}\right)^{2}$ and the unresolved component $\widetilde{N}_{s g s}=\frac{1}{2} C_{N} \frac{\nu_{t}}{\Delta^{2}} \widetilde{\xi^{\prime \prime}}$. The constant $C_{N}$ is set to a value of 42 , which was tuned to match experimental data in [8] and has given good agreement with experimental data in several other cases $[9,10]$. As there is no nitrogen present in this flame, the 16 species ARM1 chemistry was used [19]. A total of 51 points were used to discretise $\eta$-space. The mass fractions at $\eta=0$ were set to 0.255 $\mathrm{O}_{2}$ and $0.745 \mathrm{CO}_{2}$. The mass fractions at $\eta=1$ were set to match the jet composition of the flame being studied.

The composition of the fuel and oxider streams give density fields very different to those found in methane-air flames for example. The density ratio of the coflow and jet for flame A1 is around 5. The viscosity of the two streams will also be different so the local viscosity will be a function of temperature and mixture fraction. To try and capture this we have used Sutherland's law to find the viscosity as a function of temperature for the major species, $\mathrm{CO}_{2}$, $\mathrm{CO}, \mathrm{H}_{2}, \mathrm{H}_{2} \mathrm{O}$ and $\mathrm{O}_{2}$ [20]. The mixture viscosity is then found from species viscosities and mole fractions using Wilke's equation [21]. This calculation is done in the $\mathrm{CMC}$ solver to give the local conditional viscosity from which the 
unconditional viscosity can be found as $\widetilde{\mu}=\int_{0}^{1} \widetilde{\mu \mid \eta} \widetilde{\mathcal{P}}(\eta) d \eta$. The timestep was set to $\Delta t=2 \mu s$. To initialise the flames a 'burning' flamelet was generated by solving Eq. (3) with transport terms switched off and a fixed low conditional scalar dissipation rate $\left(\widetilde{N \mid \eta_{\max }}=5 s^{-1}\right)$. This flamelet was held constant across the CMC grid while a solution was established for the hot flow field. The full 3D CMC solver was then switched on and run for $15 \mathrm{~ms}$ which is equivalent to approximately 15 flow through times for the region $z<10 d$ based on a velocity of $50 \mathrm{~m} / \mathrm{s}$, which took approximately 72 hours. Averages were taken using the last $5 \mathrm{~ms}$ of data.

\section{Results and Discussion}

\subsection{Preliminary Observations}

The experimental observations of Sevault et al [3] revealed that for all the flames studied the flame stayed attached to the nozzle with extinction not observed until around $z / d=3$. With the CMC method there is a choice to be made as to the CMC boundary conditions at the inlet, i.e. what conditional averages should be specified. This is further discussed in [14]. Both fully burning flamelets and inert distributions were tried for flame A1 and with either method it was seen that the flame was predicted to lift by around $0.5 \mathrm{~d}$. As this lifting was observed even with fully burning flamelets introduced at the inlet it can be concluded that extinction is being predicted due to the very high conditional scalar dissipation rate in the CMC cells close to the nozzle. While it is possible that the model for $\widetilde{N}_{s g s}$ is not accurate this close to the nozzle, it was also observed that $\tilde{N}_{\text {res }}$ was predicted to be sufficiently high to cause extinction by itself. From this we conclude that there must 
be some element of the fluid mechanics close to the nozzle, which leads to low scalar dissipation rate in this region, that we are not capturing. This is supported by [3] who state that the squared off end of the nozzle helped to stabilise the flame. As it is unclear how to model this, we have chosen to set burning flamelets as the inlet CMC condition. We also limit $\widetilde{N \mid \eta}<30 s^{-1}$ for the region $z / d<1$ in order to account for a low scalar dissipation rate region around the nozzle.

All flames were initialised using the procedure outlined in Section 2.3 using jet composition and velocity details correct for the flame of interest before the full 3D CMC solver was started. Evidence of extinction in the form of low $O H$ values in regions with stoichiometric mixture fraction was clear in the resolved LES results for all flames. Experimental evidence of the instantaneous pattern of extinction is presented in [3] by images of the flame which have been binarized according to a threshold to reveal local extinctions. We have attempted to present our LES results in a similar manner in Fig. 1. Isosurfaces of $O H$ mass fraction at some threshold value were plotted with shading removed to produce flattened images as from the experiment. The actual value of the threshold is somewhat arbitrary but we have employed $Y_{O H}=0.00075$. As in the experiment note that the image is a line-of-sight image through the flame and so any dark areas in the image indicate that $Y_{O H}$ is less than the threshold value on both sides of the flame so there is an extinction on both sides. The images shown are instantaneous snapshots but are representative of the results for the flames. It can be seen in Fig. 1 that the location of the extinction region is correctly predicted in the region around $z / d=4$. Note that the extinction does not occur until around two to 
three diameters from the jet which is beyond the region where the conditional scalar dissipation rate has been limited. We also see that the correct trend of increasing extinction from A1 to A3 as $H_{2}$ is reduced is correctly captured.

\subsection{Conditional Statistics}

To consider the degree of extinction predicted qualitatively we will first consider the conditional averages of species. Experimental data is available for conditional averages taken at $z / d=3,5$, and 10 . These averages were compiled from statistics taken across the flame at these locations. In order to compare with these the $\mathrm{CMC}$ results have been processed to give a single conditional for each of the three axial locations. This has been done by averaging data from all CMC cells with the desired axial position in a PDF weighted manner such that

$$
\widetilde{\phi \mid \eta}^{*}=\frac{\int \widetilde{\phi \mid \eta} \widetilde{\mathcal{P}}(\eta) d V}{\int \widetilde{\mathcal{P}}(\eta) d V}
$$

The averages were taken over 26 points in time each separated by $0.2 \mathrm{~ms}$. This is equivalent to approximately five flow through times for the region $z<10 d$ based on a velocity of $50 \mathrm{~m} / \mathrm{s}$. Conditional temperature is available for comparison at $z / d=5$ which is just downstream of the main extinction region. This is plotted in Fig. 2 and the effect of increased extinction from flame A1 to A3 can clearly be seen in both experiment and simulation with very good agreement for conditional temperature for both flames.

The conditionally averaged $\mathrm{O}_{2}$ mass fraction is presented in Fig. 3. The agreement for both flames at $z / d=5$ is again very good. At $z / d=3$ it can be seen that, while the $\mathrm{CMC}$ is correctly predicting the trend of increasing 
extinction (and therefore increased $\mathrm{O}_{2}$ at stoichiometry) from A1 to A3, there is in fact more extinction predicted with the CMC than is observed experimentally. By $z / d=10$ there is relatively little extinction but at rich mixture fractions the $\mathrm{O}_{2}$ level is higher in A3 than A1 which is correctly predicted by the CMC calculation. The overprediction of extinction at $z / d=$ 3 can be observed also in the conditional averages of $\mathrm{H}_{2} \mathrm{O}$ shown in Fig. 4, where the level is seen to be lower in the CMC prediction at $z / d=3$. However the conditional average is predicted to be lower than experiment, particularly at $z / d=5$, and still to some extent by $z / d=10$. (A similar trend is seen for $C O$, and this is shown in supplementary material.) As we have already seen very good agreement for temperature and $\mathrm{O}_{2}$ at $z / d=5$ the discrepancy in $\mathrm{H}_{2} \mathrm{O}$ values at this position indicates that the the $\mathrm{H}_{2} \mathrm{O}$ level is being underpredicted at a given temperature, this suggests that details of the chemistry are not being completely captured here.

There are several possible reasons why there is an overprediction of the amount of extinction close to the nozzle and a difference in the level of agreement between $\mathrm{T}$ and $\mathrm{H}_{2} \mathrm{O}$. We have already discussed in the previous section that the conditional scalar dissipation rate might be too high close to the nozzle. The unresolved scalar dissipation rate constant was obtained using data for a range of axial locations [8] and it may not be accurate very close to a nozzle. Also as discussed in the next section the flow field is not accurately predicted in this region which will have an effect on the extinction. The modelled conditional diffusion term used here may also be a possible source of error. Sevault et al [3] discuss the likely influence of differential diffusion in this flame due to its high $\mathrm{H}_{2}$ content. This phenomenon is not 
included in the CMC formulation here which may be responsible for the differences observed from experiment. It is possible to include differential diffusion in $\mathrm{CMC}[22]$ and so its influence could be investigated in future work.

\subsection{Radial Averages}

Radial averages of resolved $\mathrm{H}_{2} \mathrm{O}$ are presented in Fig. 5. These radial averages have been produced by averaing the results from LES cells with a given axial and radial position using the same points in time used for the conditional averages. Hence radial averaging is done in both space and time. As expected, given the underprediction of the conditional values, the peak resolved values are also underpredicted although the trend with decreasing jet $H_{2}$ content is correctly reproduced. (Again, the trend for $C O$ is similar, but omitted here to save space.) At $z / d=5$ and $z / d=$ 10 it can be seen that the mixing of the jet is overpredicted, in the sense that the spreading in the radial direction is too fast, and it is possible that this excess mixing is responsible for some of the excess localised extinction observed in the calculation. Predicting the early part of a jet with LES is difficult and it it is likely that the unusual composition seen in this jet makes this more difficult. Calculating the viscosity at a given temperature for each species and then using Wilke's equation to give a mixture viscosity has made it possible to make viscosity a function of both mixture fraction and temperature. However the accuracy of applying Sutherland's law with constants for pure gases to mixture components is doubtful and this could lead to errors in the calculation of viscosity and hence to the rate at which the jet mixes out. 
The radial average of $\mathrm{O}_{2}$ is shown in Fig. 6. The difference in the level of extinction seen in Fig. 3 is again seen in the radial results, particularly at $z / d=3$ where the greatest extinction takes place. The radial extent of the region at $z / d=5$ and 3 where an increased $O_{2}$ level is seen in flame A3 is smaller in the LES results than in the experiment. This is likely to be due to the incorrect level of mixing seen in the LES results. The increased level of $\mathrm{O}_{2}$ in the centre of the A3 jet at $z / d=10$ is correctly predicted by the $\mathrm{CMC}$ and this corresponds with the increased conditional $\mathrm{O}_{2}$ seen in Fig. 3 at this position.

\subsection{Fully Burning Probability}

In order to quantify the extent of extinction seen in the flames Sevault et al [3] defined a fully burning probability or FBP. This was defined as being the probability of finding a burning compostion in a narrow band of mixture fraction centred on the mixture fraction giving highest temperature. Fully burning was defined as having $T>1700 K$. To compare with this we have calculated FBP from the CMC results. This is based on $\widetilde{T \mid \eta_{\max }}$ where $\eta_{\max }$ is the mixture fraction giving maximum temperature. A 'burning' function

$\lambda$ is defined which takes a value of 1 if $\widetilde{T \mid \eta_{\max }}>1700$ and 0 otherwise. The CMC FBP is then found by averaging

$$
F B P=\frac{\int \lambda \widetilde{\mathcal{P}}\left(\eta_{\max }\right) d V}{\int \widetilde{\mathcal{P}}\left(\eta_{\max }\right) d V}
$$

The integration is taken over all CMC cells with a given axial position from all time intervals. The results from this are compared with the experimental data in Fig. 7. We see further confirmation that the degree of extinction 
is overpredicted but that the axial location of extinction and the trend of increasing extinction with decreasing $H_{2}$ content is correctly predicted. It appears that for weak extinctions the CMC underestimates the extinction, whereas for stronger extinctions the $\mathrm{CMC}$ flamelet is predicted to become almost fully extinct and thus overestimates the extinctions.

\section{Conclusions}

An unstructured formulation of the LES-CMC method has been applied to the Oxy-fuel jet flames of [3]. The unstructured CMC allows grid resolution to be concentrated where required around the stoichiometric mixture fraction contour in the early part of the jet. By using the volume flux at the LES resolution to calculate the CMC transport terms more information about the flow field can be included in the CMC calculation. The LES-CMC calculations have been successful in capturing both the physical location of localised extinction in the flame and the trend of increasing extinction as the $\mathrm{H}_{2}$ content in the jet is reduced.

The results here also highlight the challenges of simulating flames with the different compositions of both fuel and oxidiser streams encountered in oxy-fuel flames. The extent of the extinction, as seen in conditional and unconditional mass fractions and in the fully burning probability, is seen to be

higher in the simulations than in the experiment. It is also seen that there are different levels of agreement for different chemical species, suggesting that the full details of the chemistry are not being captured. One possible explanation for the disagreement with experiment is that the current formulation neglects differential diffusion and including this would be an interesting avenue for 
future research into this flame.

\section{Acknowledgements}

Calculations were performed on HPC-Midlands funded by the UK Engi-

neering and Physical Sciences Research Council, Grant ref EP/K000063/1.

\section{References}

\section{References}

[1] M. Ditaranto, J. Sautet, J. Samaniego, Exp. Fluids 30 (2001) 253-261.

[2] M. Ditaranto, J. Hals, Combust. Flame 146 (2006) 493-512.

[3] A. Sevault, M. Dunn, R. S. Barlow, M. Ditaranto, Combust. Flame 159 (2012) 3342-3352.

[4] W. Jones, V. N. Prasad, Combust. Flame 157 (2010) 1621-1636.

[5] M. Ihme, H. Pitsch, Combust. Flame 155 (2008) 90-107.

[6] Y. Ge, M. J. Cleary, A. Y. Klimenko, Proc. Combust. Inst. 34 (2013) 1325-1332.

[7] Y. Yang, H. Wang, S. Pope, J. H. Chen, Proc. Combust. Inst. 34 (2013) $1241-1249$.

[8] A. Garmory, E. Mastorakos, Proc. Combust. Inst. 33 (2011) 1673-1680.

[9] S. Ayache, E. Mastorakos, Flow Turb. Combust. 88 (2012) 207-231. 
[10] A. Tyliszczak, D. E. Cavaliere, E. Mastorakos, Flow Turb. Combust. 92 (2014) 237-267.

[11] M. J. Cleary, J. H. Kent, Combust. Flame 143 (2005) 357-368.

[12] S. Navarro-Martinez, A. Kronenburg, F. Di Mare, Flow Turb. Combust. 75 (2005) 245-274.

[13] A. Triantafyllidis, E. Mastorakos, R. Eggels, Combust. Flame 156 (2009) 2328-2345.

[14] A. Garmory, E. Mastorakos, Int. J. Heat Fluid Fl. 39 (2013) 53-63.

[15] OpenFOAM, v. 2.1.1 (2013) www.openfoam.com.

[16] S. B. Pope, Turbulent Flows, Cambridge University Press, Cambridge, 2000.

[17] A. Triantafyllidis, E. Mastorakos, Flow Turb. Combust. 84 (2010) 481512.

[18] E. O'Brien, T.-L. Jiang, Phys. Fluids 3 (1991) 3121-3123.

[19] C. J. Sung, C. K. Law, J. Y. Chen, Proc. Combust. Inst. 27 (1998) 295-304.

[20] F. M. White, Viscous Fluid Flow, McGraw-Hill, New York, 1991.

[21] C. R. Wilke, J. Chem. Phys. 18 (1950) 517-519.

[22] A. Kronenburg, R. W. Bilger, Combust. Sci. Tech. 166 (2001) 195-227. 


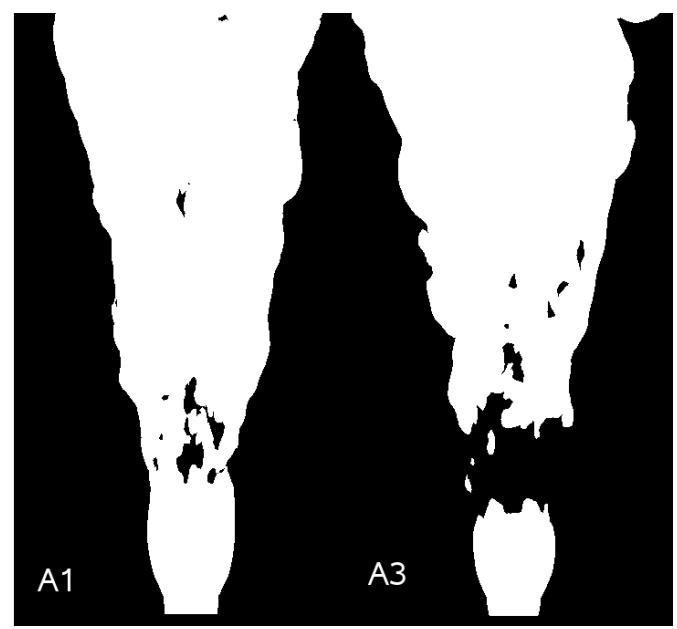

Figure 1: Predicted LES instantaneous isosurfaces of threshold $O H$ mass fraction for flames A1 and A3. Dark areas represent extinction on both sides of the flame.

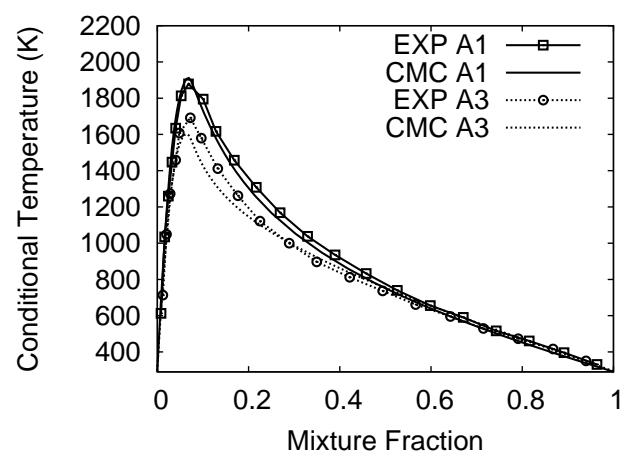

Figure 2: Mean conditional temperature at $z / d=5$ for flames A1 and A3 from experiment [3] and CMC calculation. 

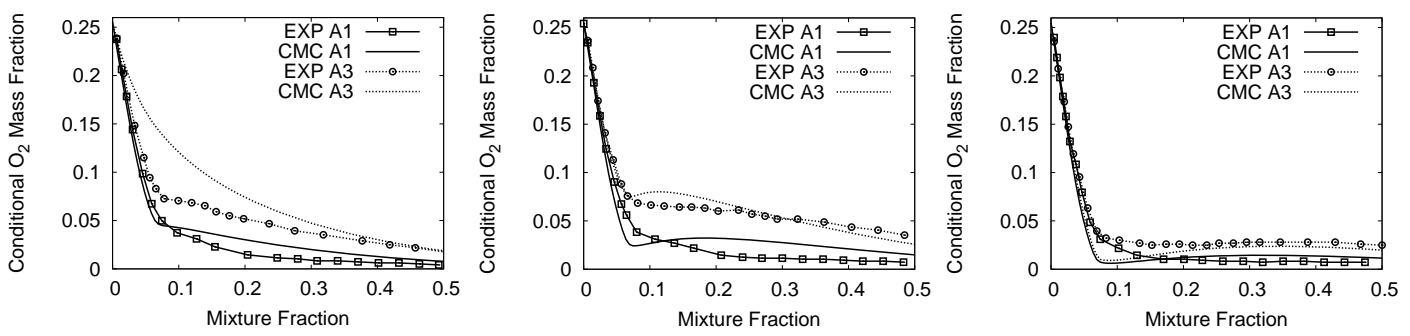

Figure 3: Mean conditional $\mathrm{O}_{2}$ mass fraction at $z / d=3$ (left), $z / d=5$ (middle) and $z / d=10$ (right) for flames A1 and A3 from experiment [3] and CMC calculation.
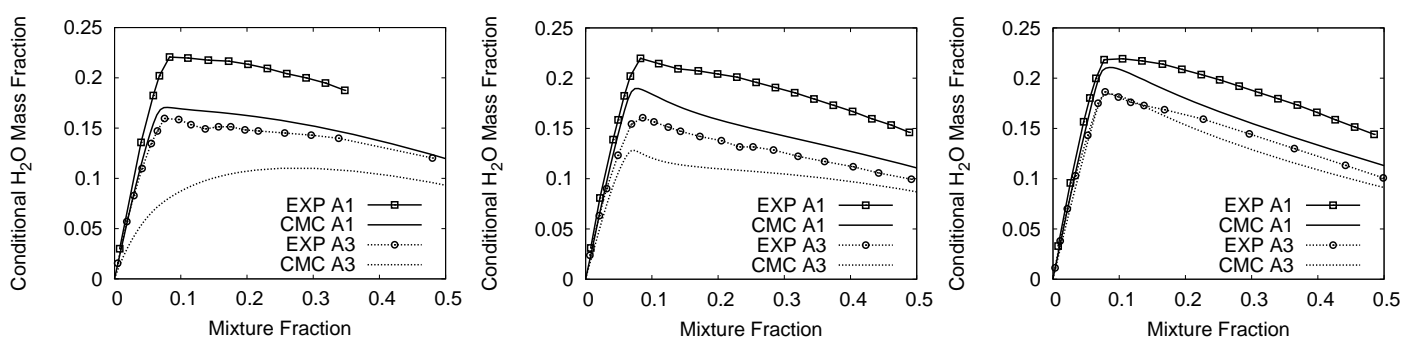

Figure 4: Mean conditional $\mathrm{H}_{2} \mathrm{O}$ mass fraction at $z / d=3$ (left), $z / d=5$ (middle) and $z / d=10$ (right) for flames A1 and A3 from experiment [3] and CMC calculation.
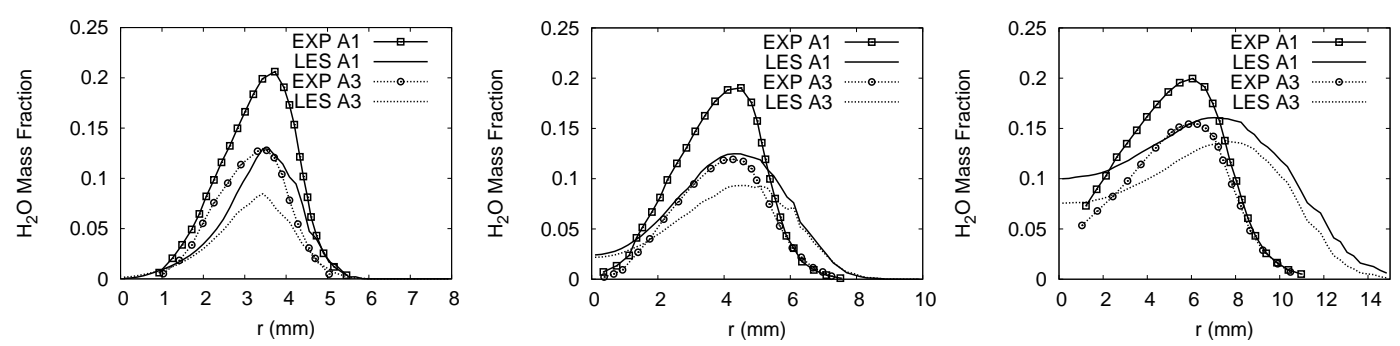

Figure 5: Radially averaged $\mathrm{H}_{2} \mathrm{O}$ mass fraction at $z / d=3$ (left), $z / d=5$ (middle) and $z / d=10$ (right) for flames A1 and A3 from experiment [3] and LES calculation. 

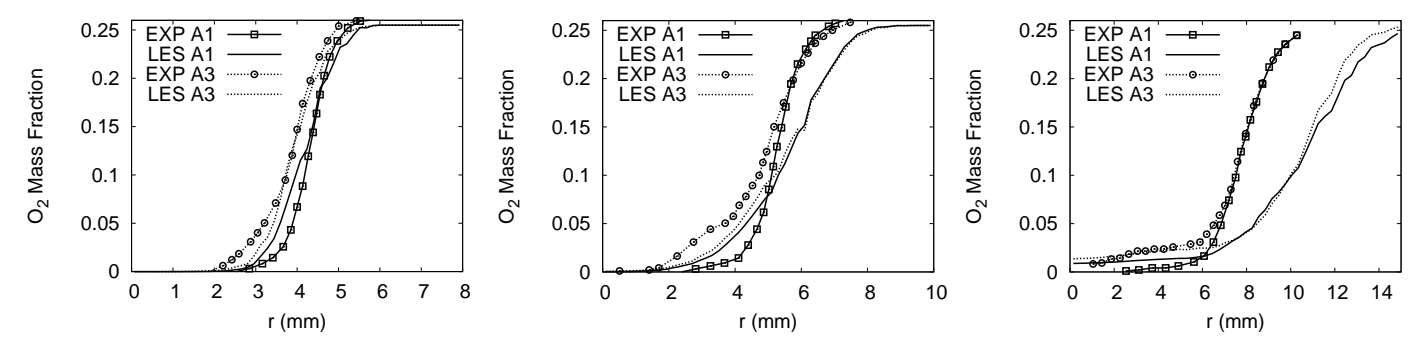

Figure 6: Radially averaged $\mathrm{O}_{2}$ mass fraction at $z / d=3$ (left), $z / d=5$ (middle) and $z / d=10$ (right) for flames A1 and A3 from experiment [3] and LES calculation.

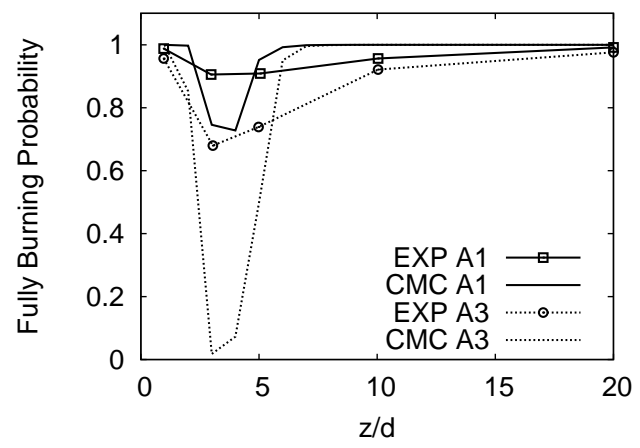

Figure 7: Fully Burning Probability against axial position from experiment [3] and CMC results 